\title{
Microlensing planet detection via geosynchronous and low Earth orbit satellites
}

\author{
F. Mogavero and J. P. Beaulieu
}

\begin{abstract}
Sorbonne Universités, UPMC Paris 6 et CNRS, UMR 7095, Institut d'Astrophysique de Paris, 98bis bd Arago, 75014 Paris, France e-mail: [mogavero; beaulieu] @iap.fr
\end{abstract}

Received 16 September 2015 / Accepted 16 October 2015

\begin{abstract}
Planet detection through microlensing is usually limited by a well-known degeneracy in the Einstein timescale $t_{\mathrm{E}}$, which prevents mass and distance of the lens to be univocally determined. It has been shown that a satellite in geosynchronous orbit could provide masses and distances for most standard planetary events $\left(t_{\mathrm{E}} \approx 20\right.$ days) via a microlens parallax measurement. This paper extends the analysis to shorter Einstein timescales, $t_{\mathrm{E}} \approx 1$ day, when dealing with the case of Jupiter-mass lenses. We then study the capabilities of a low Earth orbit satellite on even shorter timescales, $t_{\mathrm{E}} \approx 0.1$ days. A Fisher matrix analysis is employed to predict how the 1- $\sigma$ error on parallax depends on $t_{\mathrm{E}}$ and the peak magnification of the microlensing event. It is shown that a geosynchronous satellite could detect parallaxes for Jupiter-mass free floaters and discover planetary systems around very low-mass brown dwarfs. Moreover, a low Earth orbit satellite could lead to the discovery of Earth-mass free-floating planets. Limitations to these results can be the strong requirements on the photometry, the effects of blending, and in the case of the low orbit, the Earth's umbra.
\end{abstract}

Key words. gravitational lensing: micro - parallaxes - planets and satellites: detection - brown dwarfs

\section{Introduction}

The fundamental quantity that is routinely measured in a microlensing event is the Einstein timescale $t_{\mathrm{E}}$,

$t_{\mathrm{E}}=1.1$ days $\left(\frac{M}{M_{\mathrm{Jup}}}\right)^{1 / 2}\left(\frac{x(1-x)}{0.25}\right)^{1 / 2}\left(\frac{D_{\mathrm{S}}}{8 \mathrm{kpc}}\right)^{1 / 2}\left(\frac{200 \mathrm{~km} \mathrm{~s}^{-1}}{V}\right)$

where $x \equiv D_{\mathrm{L}} / D_{\mathrm{S}}$. It depends on the lens mass $M$, the lens and source distances from Earth $D_{\mathrm{L}}$ and $D_{\mathrm{S}}$, and the lens-source relative velocity $V$. Consequently, the lens mass cannot be inferred without any knowledge of $x$ and $V$. This degeneracy in $t_{\mathrm{E}}$ can be resolved via the equation $M=\theta_{\mathrm{E}} / \kappa \pi_{\mathrm{E}}$, with $\kappa$ a constant, if the angular Einstein radius $\theta_{\mathrm{E}}=t_{\mathrm{E}} V / D_{\mathrm{L}}$ and the microlens parallax $\pi_{\mathrm{E}}=1 \mathrm{AU}\left(D_{\mathrm{L}}^{-1}-D_{\mathrm{S}}^{-1}\right) / \theta_{\mathrm{E}}$

$\pi_{\mathrm{E}}=4.3\left(\frac{1 \text { day }}{t_{\mathrm{E}}}\right)\left(\frac{1-x}{0.5}\right)\left(\frac{200 \mathrm{~km} \mathrm{~s}^{-1}}{V}\right)$

can both be measured from the event light curve (Gould 2013, from now on G13). The quantity $\theta_{\mathrm{E}}$ is measured via finite source effects in high-magnification single-lens events and in a large number of the planetary ones. G13 has shown that for planetary events $\left(t_{\mathrm{E}} \approx 20\right.$ days) with peak amplifications $A_{\max } \gtrsim$ 20 , a satellite placed in geosynchronous orbit would measure the microlens parallax $\pi_{\mathrm{E}}$, providing lens masses. This result is especially important in the perspective of the Wide Field Infrared Space Telescope (WFIRST) mission (Barry et al. 2011): a geosynchronous orbit is currently a strong alternative to the second Lagrangian point (L2) (Spergel et al. 2015). In particular, G13 studies the regime where the satellite orbital period $P$ is much shorter than the shortest timescale of the microlens event, $t_{\mathrm{eff}} \equiv \beta t_{\mathrm{E}}$, with $\beta$ the lens-source impact parameter in units of $\theta_{\mathrm{E}}$. The following scaling law for the relative error on $\pi_{\mathrm{E}}$ is predicted:

$\frac{\sigma_{\pi_{\mathrm{E}}}}{\pi_{\mathrm{E}}} \propto t_{\mathrm{E}}^{1 / 2} \beta R^{-1}, \quad P \ll \beta t_{\mathrm{E}}$ and $\beta \ll 1$

where $R$ is the satellite orbital radius. The sensitivity to $\pi_{\mathrm{E}}$ increases towards shorter Einstein timescales. However, Eq. (3) does not apply when $P \gtrsim \beta t_{\mathrm{E}}$, as correlations between $\pi_{\mathrm{E}}$ and the other model parameters, such as $\beta$ and $t_{\mathrm{E}}$, start to affect the error on $\pi_{\mathrm{E}}$.

In this paper, after briefly setting up the Fisher matrix analysis in Sect. 2, we extend the analysis of G13 to shorter Einstein timescales in Sect. 3, to cover events raised by Jupiter-mass lenses $\left(t_{\mathrm{E}} \approx 1\right.$ day). Then, in Sect. 4 we study the capabilities of a low Earth orbit satellite to detect microlens parallax in case of Earth-mass lenses $\left(t_{\mathrm{E}} \approx 0.1\right.$ days $)$.

\section{Fisher matrix analysis}

The Fisher information matrix provides a way to predict, in a Bayesian framework, constraints on the best fit of a theoretical model to observational data. It allows the covariance matrix of the model parameters to be estimated via their posterior probability distribution (Sellentin et al. 2014). The Fisher matrix is a fast, analytical alternative to time-consuming methods such as the Markov-Chain Monte Carlo, even though its reliability is not always guaranteed (Vallisneri 2008). flux $F$,

The physical observable of a microlensing event is the light

$F=F_{\mathrm{s}} A+F_{\mathrm{b}}=\bar{F}[(1-v) A+v]$

where $F_{\mathrm{s}}, F_{\mathrm{b}}$, and $\bar{F} \equiv F_{\mathrm{s}}+F_{\mathrm{b}}$ are the source, blending, and baseline fluxes, and $v \equiv F_{\mathrm{b}} /\left(F_{\mathrm{s}}+F_{\mathrm{b}}\right)$ defines the blending ratio. In a 
point source-point lens (PSPL) model, the amplification factor $A$ is given by $A(u)=\left(u^{2}+2\right) / u \sqrt{u^{2}+4}$, where $u$ is the magnitude of the lens-source separation vector $\boldsymbol{u}$ as seen by the observer. For an inertial observer, $u=\sqrt{\tau^{2}+\beta^{2}}$, where $\tau \equiv\left(t-t_{0}\right) / t_{\mathrm{E}}$ and $t_{0}$ is the peak time. The inertial observer model is thus described by five parameters: $\boldsymbol{\theta}=\left(\bar{F}, v, t_{0}, t_{\mathrm{E}}, \beta\right)$.

Under the assumption of Gaussian errors, the Fisher matrix elements are given by (Sellentin et al. 2014)

$\mathcal{F}_{i j}=\sum_{k=1}^{N} \frac{1}{\sigma_{k}^{2}} \frac{\partial F_{k}}{\partial \theta_{i}} \frac{\partial F_{k}}{\partial \theta_{j}}, \quad \sigma^{2}=\sigma_{0}^{2}[(1-v) A+v]$

where the index $k$ spans the set of $N$ independent observations, and $\sigma$ is the flux error. We have assumed Poisson noise-limited photometry with $\sigma_{0} \simeq(\ln 10 / 2.5) \bar{F} \sigma_{\mathrm{m}}$, where $\sigma_{\mathrm{m}}$ is the magnitude error at the light curve baseline. In the case of continuous observations, i.e. $f \beta t_{\mathrm{E}} \gg 1$, where $f$ is the number of observations per unit time, the sum appearing in Eq. (5) can be approximate by an integral over time,

$\sum_{k=1}^{N} \longrightarrow f t_{\mathrm{E}} \int_{-\infty}^{+\infty} \mathrm{d} \tau$

Since the parallax signal contributing to the matrix $\mathcal{F}$ comes from a few $t_{\text {eff }}$ near $\tau=0$, the boundaries of integration can be safely extended to infinity, as shown in Eq. (6), if the observations last more than a few event timescales ${ }^{1}$. Once the Fisher matrix is computed, the covariance matrix of the model parameters is given by its inverse, $\mathcal{F}^{-1}$ (Sellentin et al. 2014).

\subsection{Parallax effect for an Earth orbit satellite}

Observations from an Earth orbit satellite are affected by the parallax effect of its non-inertial motion, in the same way as for a telescope on Earth (Hardy \& Walker 1995). We thus consider an observatory in circular orbit around Earth with radius $R$ and period $P(\omega=2 \pi / P$ as its angular velocity). If $\lambda$ denotes the latitude of the source star with respect to the plane of this orbit, the projection of the satellite trajectory onto the plane of the sky is an ellipse with semimajor and semiminor axes $R$ and $R \sin (\lambda)$, respectively. Following G13, one then defines $\epsilon_{\|}=\epsilon \equiv R / 1 \mathrm{AU}$ and $\epsilon_{\perp} \equiv R \sin (\lambda) / 1$ AU. Let $\theta$ denote the direction of the lenssource relative motion in the plane of the sky with respect to the projected major axis of the satellite orbit. The lens-source separation vector seen by the satellite is given by $\boldsymbol{u}=(\tau \cos \theta-$ $\left.\beta \sin \theta+\epsilon_{\|} \pi_{\mathrm{E}} \cos \left(\omega t_{\mathrm{E}} \tau+\varphi\right), \tau \sin \theta+\beta \cos \theta+\epsilon_{\perp} \pi_{\mathrm{E}} \sin \left(\omega t_{\mathrm{E}} \tau+\varphi\right)\right)$, where $\varphi$ is the satellite orbital phase with respect to the peak time $t_{0}$ (Hardy \& Walker 1995, G13). If one introduces the microlens parallax vector $\pi_{\mathbf{E}}=\left(\pi_{\|}, \pi_{\perp}\right)=\pi_{\mathrm{E}}(\cos \theta, \sin \theta)$, the non-inertial observer model is described by seven parameters: $\boldsymbol{\theta}=\left(\bar{F}, v, t_{0}, t_{\mathrm{E}}, \beta, \pi_{\|}, \pi_{\perp}\right)$. The two additional flux derivatives $\partial F / \partial \pi_{\|}$and $\partial F / \partial \pi_{\perp}$ appearing in the Fisher matrix (5) are proportional to $\partial u / \partial \pi_{\|}$and $\partial u / \partial \pi_{\perp}$, respectively, with

$$
\begin{aligned}
& u \frac{\partial u}{\partial \pi_{\|}} \simeq \epsilon_{\|} \tau \cos \left(\omega t_{\mathrm{E}} \tau+\varphi\right)+\epsilon_{\perp} \beta \sin \left(\omega t_{\mathrm{E}} \tau+\varphi\right), \\
& u \frac{\partial u}{\partial \pi_{\perp}} \simeq-\epsilon_{\|} \beta \cos \left(\omega t_{\mathrm{E}} \tau+\varphi\right)+\epsilon_{\perp} \tau \sin \left(\omega t_{\mathrm{E}} \tau+\varphi\right) .
\end{aligned}
$$

\footnotetext{
1 Substitution (6) causes the element $\mathcal{F}_{\bar{F} \bar{F}}$ to diverge, meaning that an arbitrary precision on $\bar{F}$ can be attained by observing enough in the baseline of the light curve. The parameter $\bar{F}$ can thus be safely considered as a constant and can be removed from the Fisher analysis.
}

The approximate equalities mean we neglect terms that are linear in $\pi_{\mathrm{E}}$. Moreover, one can assume $u \simeq \sqrt{\tau^{2}+\beta^{2}}$. These approximations are justified as long as $\epsilon \pi_{\mathrm{E}} \beta^{-1} \ll 1$. In the following analysis, the above inequality is safely verified. G13 also assumes its validity.

\subsection{Sensitivity to $\pi_{\mathrm{E}}$}

Once the Gaussian approximation to the posterior probability distribution of $\pi_{\|}$and $\pi_{\perp}$ is known via the Fisher matrix, one can forecast the sensitivity of microlensing observations to $\pi_{\mathrm{E}}$ through standard error propagation. Since small variations in the parallax parameters are related by $\delta \pi_{\mathrm{E}} \simeq \cos \theta \delta \pi_{\|}+\sin \theta \delta \pi_{\perp}$, the $1-\sigma$ error on $\pi_{\mathrm{E}}$ is given by the equation

$\sigma_{\pi_{\mathrm{E}}}^{2}=\cos ^{2}(\theta) \sigma_{\pi_{\|}}^{2}+\sin ^{2}(\theta) \sigma_{\pi_{\perp}}^{2}+\sin (2 \theta) \operatorname{cov}\left(\pi_{\|}, \pi_{\perp}\right)$,

where $\operatorname{cov}\left(\pi_{\|}, \pi_{\perp}\right)$ is the covariance between the two parallax parameters. In principle, $\sigma_{\pi_{\mathrm{E}}}^{2}$ depends on both $\theta$ and $\varphi$.

To present results that are independent of the geometry of the event, we summarise the information contained into Eq. (8) by finding the extrema of $\sigma_{\pi_{\mathrm{E}}}^{2}(\theta, \varphi)$ over $\theta, \varphi \in[0,2 \pi]$. To do this, one first notices that, according to Eq. (7), $\sigma_{\pi_{\|}}^{2}, \sigma_{\pi_{\perp}}^{2}$, and $\operatorname{cov}\left(\pi_{\|}, \pi_{\perp}\right)$ do not depend on $\theta$, but only on $\varphi$. One can thus analytically find the extrema of $\sigma_{\pi_{\mathrm{E}}}^{2}(\theta, \varphi)$ over the $\theta$ range,

$\sigma_{\pi_{\mathrm{E}}, \pm}^{2}(\varphi)=\frac{\sigma_{\pi_{\|}}^{2}+\sigma_{\pi_{\perp}}^{2}}{2} \pm \frac{\sqrt{\left(\sigma_{\pi_{\|}}^{2}-\sigma_{\pi_{\perp}}^{2}\right)^{2}+4 \operatorname{cov}\left(\pi_{\|}, \pi_{\perp}\right)^{2}}}{2}$

where the plus and minus signs stand for the maxima and minima. The values of $\theta$ that correspond to these extrema are given by $\tan (2 \theta)=2 \operatorname{cov}\left(\pi_{\|}, \pi_{\perp}\right) /\left(\sigma_{\pi_{\|}}^{2}-\sigma_{\pi_{\perp}}^{2}\right)^{2}$. Then, one can find the extrema of Eq. (9) over the $\varphi$ range numerically, and they only depend on $t_{\mathrm{E}}$ and $\beta$ :

$\sigma_{\pi_{\mathrm{E}}, \max }^{2} \equiv \max _{\varphi \in[0,2 \pi]} \sigma_{\pi_{\mathrm{E}},+}^{2}(\varphi), \quad \sigma_{\pi_{\mathrm{E}}, \min }^{2} \equiv \min _{\varphi \in[0,2 \pi]} \sigma_{\pi_{\mathrm{E}},-}^{2}(\varphi)$.

We note that for $P \ll \beta t_{\mathrm{E}}$, the covariance between $\pi_{\|}$and $\pi_{\perp}$ vanishes, $\sigma_{\pi_{\|}}^{2}$ and $\sigma_{\pi_{\perp}}^{2}$ become independent of $\varphi$, and the extrema of $\sigma_{\pi_{\mathrm{E}}}^{2}(\theta, \varphi)$ are simply given by $\max \left(\sigma_{\pi_{\|}}^{2}, \sigma_{\pi_{\perp}}^{2}\right)$ and $\min \left(\sigma_{\pi_{\|}}^{2}, \sigma_{\pi_{\perp}}^{2}\right)$.

\section{Geosynchronous orbit satellite}

We then consider the case of a geosynchronous orbit (GSO) satellite, orbiting in the equatorial plane and targeting the Galaxy bulge, $P=23 \mathrm{~h} 56 \mathrm{~min} 4 \mathrm{~s}, R=6.6 R_{\oplus}, \lambda=30^{\circ}$. We assume $f=1$ observation $/ 3 \mathrm{~min}$, which is reasonable for a WFIRSTlike satellite (G13) and a magnitude error at baseline $\sigma_{\mathrm{m}}=0.01$. Figure 1 shows the predicted relative error $\sigma_{\pi_{\mathrm{E}}} / \pi_{\mathrm{E}}$ as a function of the Einstein timescale $t_{\mathrm{E}}$, for zero blending $(v=0)$. Since, from Eq. (2), $\pi_{\mathrm{E}}$ depends on $D_{\mathrm{L}}, D_{\mathrm{S}}$, and $V$, we consider a typical disk lens at $D_{\mathrm{L}}=4 \mathrm{kpc}$ and $V=200 \mathrm{~km} \mathrm{~s}^{-1}\left(D_{\mathrm{S}}=8 \mathrm{kpc}\right.$ for a source in the bulge of the Galaxy).

For an impact parameter $\beta=0.1$, which corresponds to a peak amplification $A_{\max } \approx 10$, we plot the region $\sigma_{\pi_{\mathrm{E}} \text {, min }} \leq$ $\sigma_{\pi_{\mathrm{E}}} \leq \sigma_{\pi_{\mathrm{E}} \text {, max }}$ to take the variations induced by the parameters $\varphi$ and $\theta$ into account. Figure 1 clearly shows two different

2 The extrema $\sigma_{\pi_{\mathrm{E}}, \pm}(\varphi)$ correspond to a lens-source relative motion aligned with one of the principal axes of the bivariate Gaussian distribution which approximates the posterior probability distribution of $\pi_{\|}$and $\pi_{\perp}$ in the Fisher matrix analysis, when one marginalises over the remaining parameters. 
F. Mogavero and J. P. Beaulieu: Microlensing planet detection via geosynchronous and low Earth orbit satellites

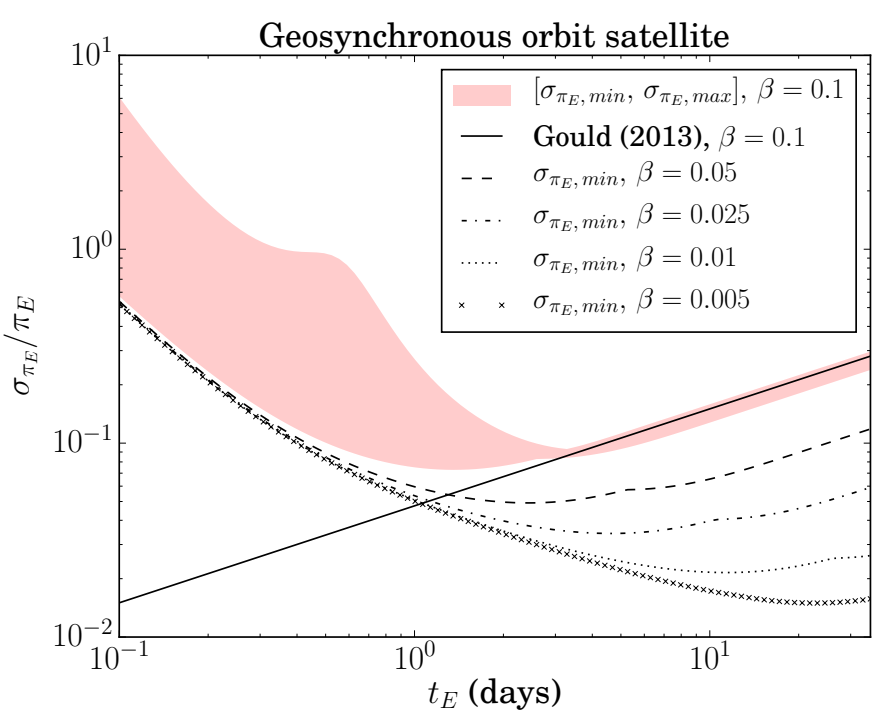

Fig. 1. Relative error on $\pi_{\mathrm{E}}$ plotted against $t_{\mathrm{E}}$ for a GSO satellite. We assume $3 \mathrm{~min}$ exposures, $\sigma_{\mathrm{m}}=0.01$, and zero blending. For $\beta=0.1$ the region $\sigma_{\pi_{\mathrm{E}}} / \pi_{\mathrm{E}} \in\left[\sigma_{\pi_{\mathrm{E}}, \min }, \sigma_{\pi_{\mathrm{E}}, \max }\right] / \pi_{\mathrm{E}}$ is shown, along with the G13 prediction. For $\beta=0.05,0.025,0.01$, and 0.005 , the minimum relative error $\sigma_{\pi_{\mathrm{E}}, \min } / \pi_{\mathrm{E}}$ is plotted.

regimes. For $t_{\mathrm{E}} \gg 1$ day, the relative error on $\pi_{\mathrm{E}}$ scales as $t_{\mathrm{E}}^{1 / 2}$, in agreement with Eq. (3), the analytic prediction of G13. For $t_{\mathrm{E}} \ll 1$ day, the relative error increases by decreasing $t_{\mathrm{E}}$, as a result of the correlations between $\pi_{\|}, \pi_{\perp}$, and the other parameters $v, t_{0}, t_{\mathrm{E}}$, and $\beta$. In the intermediate region 1 day $\leq t_{\mathrm{E}} \leq$ 3 days, a maximum sensitivity to $\pi_{\mathrm{E}}$ is attained. In particular, at $t_{\mathrm{E}} \approx 3$ days, the error range is very narrow around $\sigma_{\pi_{\mathrm{E}}} / \pi_{\mathrm{E}} \approx 9 \%$. By decreasing $t_{\mathrm{E}}, \sigma_{\pi_{\mathrm{E}} \text {, max }}$ increases steeply, while $\sigma_{\pi_{\mathrm{E}} \text {, min }}$ still decreases to a minimum value of about $7 \%$ at $t_{\mathrm{E}} \approx 1$ day. This means that one can end up with a good sensitivity to $\pi_{\mathrm{E}}$ even at $t_{\mathrm{E}} \approx 1$ day, where the maximum relative error is already a steep function of the Einstein timescale.

For smaller impact parameters, $\beta<0.1$, Fig. 1 shows the minimum relative error $\sigma_{\pi_{\mathrm{E}} \text {, min }} / \pi_{\mathrm{E}}$. The region of maximum sensitivity to $\pi_{\mathrm{E}}$ clearly moves to higher Einstein timescales. If one considers the Einstein timescale $t_{\mathrm{E}}^{\star}$ which minimizes $\sigma_{\pi_{\mathrm{E}}, \min } / \pi_{\mathrm{E}}$, it approximately scales according to

$t_{\mathrm{E}}^{\star} \propto P \beta^{-1}$.

Indeed, it separates the two regimes $\beta t_{\mathrm{E}} \ll P$ and $\beta t_{\mathrm{E}} \gg P$. To estimate the minimum relative error for a given $\beta$, one can write that, approximately, $\sigma_{\pi_{\mathrm{E}}, \min } / \pi_{\mathrm{E}} \propto t_{\mathrm{E}}^{1 / 2} \beta R^{-1} g\left(\beta t_{\mathrm{E}} \omega\right)$, where $g(y)$ is a function that tends to 1 for $y \rightarrow \infty$, to match Eq. (3). Since Eq. (11) implies $\beta t_{\mathrm{E}}^{\star} \omega=$ const, one obtains

$\frac{\sigma_{\pi_{\mathrm{E}}, \min }}{\pi_{\mathrm{E}}}\left(t_{\mathrm{E}}^{\star}\right) \propto P^{1 / 2} \beta^{1 / 2} R^{-1}$.

Equations (11) and (12) are in good agreement with Fig. 1. The maximum sensitivity to $\pi_{\mathrm{E}}$ increases slowly by decreasing the impact parameters $\beta$, while the range of the Einstein timescales that allow for a parallax detection clearly widens.

From the above analysis and Eq. (1), it follows that a GSO satellite is naturally optimized to measure microlens parallax $\pi_{\mathrm{E}}$ in events raised by free-floating objects with masses spanning from a fraction to a few dozens $M_{\text {Jup }}$. The peak amplifications for such a measure can be as low as 10 , or even somewhat lower for closer lenses. If, additionally, the Einstein angle $\theta_{\mathrm{E}}$ can be

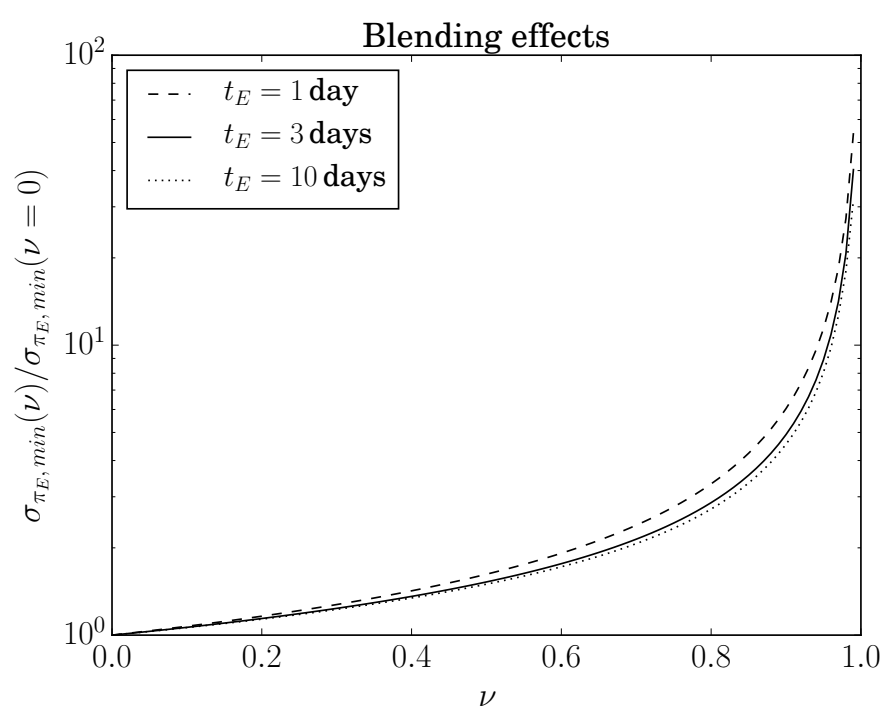

Fig. 2. Ratio $\sigma_{\pi_{\mathrm{E}}, \min }(v) / \sigma_{\pi_{\mathrm{E}}, \min }(v=0)$ against $v$ for a GSO satellite and $\beta=0.1$, with the same assumptions as in Fig. 1. Three Einstein timescales are shown: $t_{\mathrm{E}}=1,3$, and 10 . Clearly the error diverges for $v \rightarrow 1$.

measured via finite source effects (starting at $A_{\max } \approx 50$ for a Jupiter-mass lens and a Sun-like source, see Eq. (14)), the lens mass can be inferred for this class of events.

Analogously, since for a bulge source the Einstein radius scales as $R_{\mathrm{E}}=t_{\mathrm{E}} V=0.12 \mathrm{AU}\left(M / M_{\mathrm{Jup}}\right)^{1 / 2}(x(1-x) / 0.25)^{1 / 2}$, a GSO satellite is naturally optimized to discover planets in tight orbits around low-mass brown dwarfs ${ }^{3}$. In particular, as a matter of speculation, it could lead to the discovery of miniature planetary systems around planetary-mass brown dwarfs ${ }^{4}$. In fact, disks have been found in the past fifteen years to surround brown dwarfs with masses in the range 5-15 $M_{\text {Jup }}$ (see, for example, Luhman et al. 2005b,a). In particular, the disk around OTS 44 has been estimated at roughly $30 M_{\oplus}$ (Joergens et al. 2013). The question of whether planets can form out of such disks naturally arises, but very little is known about these hypothetical planetary systems, even from a theoretical point of view. Gravitational microlensing could thus lead to fundamental discoveries in this field.

\subsection{Blending}

G13 adopts $\sigma^{2}=\sigma_{0}^{2} A$ in Eq. (5), meaning that it neglects the blending flux $F_{\mathrm{b}}$ by stating that one is "only concerned with the scaling of the errors when the source is relatively highly magnified". Actually, Eq. (4) shows that the information contained in the Fisher matrix thanks to the source flux amplification is scaled by a factor $1-v$; i.e., the light curve provides less information about the model parameters if the blending factor $v$ is bigger. This is illustrated in Fig. 2, where we plot the ratio $\sigma_{\pi_{\mathrm{E}}, \min }(v) / \sigma_{\pi_{\mathrm{E}}, \min }(v=0)$ against $v$ for $\beta=0.1$ and different values of the Einstein timescale $t_{\mathrm{E}}$. The error roughly doubles at $v \approx 0.6$, and the detection of $\pi_{\mathrm{E}}$ becomes quite hopeless for $v \gtrsim 0.9$. Blending can thus highly affect the sensitivity to $\pi_{\mathrm{E}}$ and it must always be taken into account in survey planning. It is

3 Han et al. (2013) report the discovery of such a system. However, the mass ratio of this binary appears too high to envisage that the companion formed in a protoplanetary disk around the host.

4 These could also be interpreted as free-floating planet-moon systems (Bennett et al. 2014). 


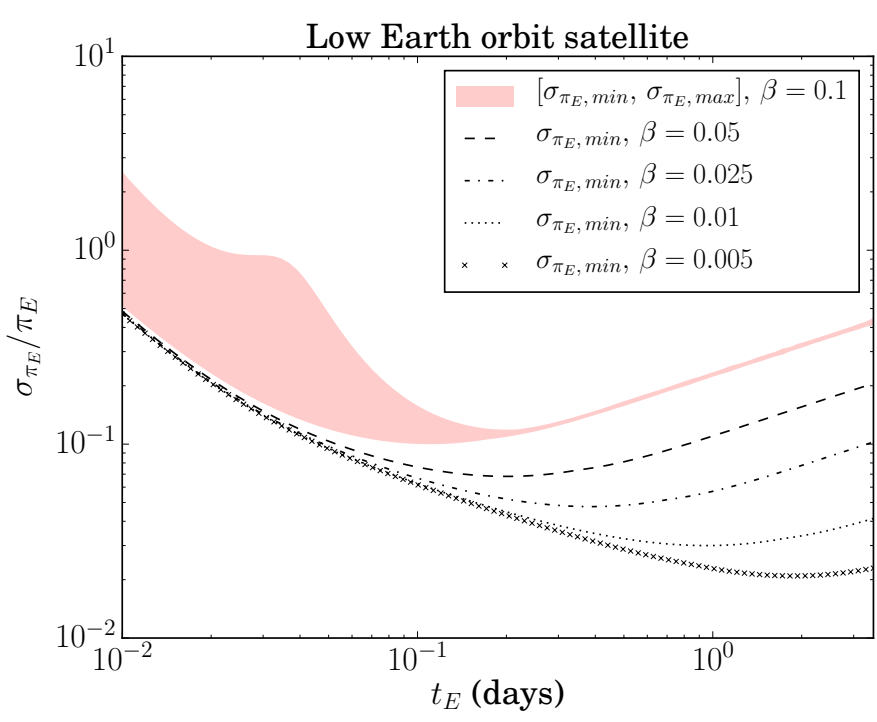

Fig. 3. Relative error on $\pi_{\mathrm{E}}$ plotted against $t_{\mathrm{E}}$ for a LEO satellite at $550 \mathrm{~km}$ above the Earth's surface. We assume $3 \mathrm{~min}$ exposures, $\sigma_{\mathrm{m}}=0.01$, and zero blending. For $\beta=0.1$ the region $\sigma_{\pi_{\mathrm{E}}} / \pi_{\mathrm{E}} \in$ $\left[\sigma_{\pi_{\mathrm{E}}, \min }, \sigma_{\pi_{\mathrm{E}}, \max }\right] / \pi_{\mathrm{E}}$ is shown. For $\beta=0.05,0.025,0.01$, and 0.005 , the minimum relative error $\sigma_{\pi_{\mathrm{E}}, \min } / \pi_{\mathrm{E}}$ is plotted.

also important to point out that, even for very low values of the blending factor, $v \rightarrow 0$, this parameter still affects the sensitivity to the other ones. Indeed, a small variation $\delta v$ produces a change in the total flux $F$ according to $|\delta F| / F \simeq(A-1) \delta v /[(1-v) A+v]$. For $v \rightarrow 0$ and $A \gg 1$, one obtains $|\delta F| / F \simeq \delta v$, which means that the contribution of $v$ to the Fisher matrix is different from zero even for vanishing blending factors. Gould's assumption in G13 is only valid for $v \rightarrow 0$ and $P \ll \beta t_{\mathrm{E}}$, because in this regime the correlations between $v$ and the parallax parameters $\pi_{\|}$and $\pi_{\perp}$ turn out to vanish ${ }^{5}$.

\section{Low Earth orbit satellite}

Using a space telescope in low Earth orbit (LEO), such as the Hubble Space Telescope (HST), measuring microlens parallaxes was first proposed by Honma (1999). In G13 Gould argues that this would generally be useless, since the corresponding $\epsilon=R / 1 \mathrm{AU}$ is too small. However, substituting Kepler's third law, $P^{2} \propto R^{3}$, in Eq. (12) leads to the following scaling law:

$$
\frac{\sigma_{\pi_{\mathrm{E}}, \min }}{\pi_{\mathrm{E}}}\left(t_{\mathrm{E}}^{\star}\right) \propto \beta^{1 / 2} R^{-1 / 4}
$$

The maximum sensitivity to $\pi_{\mathrm{E}}$ decreases slowly when one reduces the orbit radius, and what changes between a GSO and a LEO is just a factor of $(6.6)^{1 / 4} \approx 1.6$. Moreover, Eqs. (11) and (1) show that the timescale at which a LEO satellite is most sensitive to $\pi_{\mathrm{E}}$ coincides with the typical Einstein timescale of an Earth-mass object.

These properties are clearly shown in Fig. 3, where we consider a LEO satellite with HST-like orbital parameters. We assume an orbit radius $R=R_{\oplus}+550 \mathrm{~km}(P=1 \mathrm{~h} 35.5 \mathrm{~min})$ and an event latitude $\lambda=60^{\circ}$ for a bulge source, since the Hubble inclination above the equatorial plane is $28.5^{\circ}$. The predicted relative error $\sigma_{\pi_{\mathrm{E}}} / \pi_{\mathrm{E}}$ is plotted against the Einstein timescale $t_{\mathrm{E}}$ for

\footnotetext{
5 We note that, if for a given event $v=0$ holds exactly, the parameter $v$ must be removed from the corresponding Fisher analysis, and the present predictions provide an upper bound to $\sigma_{\pi_{\mathrm{E}}}$.
}

$D_{\mathrm{L}}=4 \mathrm{kpc}, V=200 \mathrm{~km} \mathrm{~s}^{-1}$, and zero blending $(v=0)$. We assume $f=1$ observation $/ 3 \mathrm{~min}$ and $\sigma_{\mathrm{m}}=0.01$ as in Fig. 1 , to allow for a quick comparison with the GSO. The plot shows the same physics as in Fig. 1, with two different regimes that arise for each choice of $\beta$. At $\beta=0.1$, the maximum sensitivity to $\pi_{\mathrm{E}}$ corresponds to Einstein timescales of one to several hours. According to Eq. (1), these $t_{\mathrm{E}}$ are typical of lens masses ranging from $M_{\oplus}$ to the super-Earth/ice giant transition. With a minimum relative error of roughly 10\%, Fig. 3 suggests that a detection of $\pi_{\mathrm{E}}$ for these lenses should be possible for $\beta \lesssim 0.1-0.2$. Since finite source effects easily arise for these kinds of lenses (see Sect. 4.1) and can provide a measure of $\theta_{\mathrm{E}}$, a LEO survey satellite could discover populations of free-floating objects ranging from terrestrial planets to super-Earths and ice giants. This would be a fantastic prospective for microlensing and exoplanet science in general. Clearly, a LEO survey would be affected by blending limitations, similar to the GSO. Moreover, the effects of Earth umbra have to be taken into account, because it reduces the fraction of the orbital period available to follow the source star (see Sect. 4.2).

For $\beta<0.1$, Eqs. (11) and (12) are consistent with Fig. 3, similar to what is obtained for the GSO. In particular, we point out that with peak amplifications of several dozen, a LEO satellite should also be able to measure microlens parallax for $t_{\mathrm{E}} \approx$ 1 day events, especially if ground-based observations are also available.

\subsection{Finite source effects}

Finite source effects arise when the impact parameter $\beta$ is comparable to $\rho=R_{\mathrm{S}} x / R_{\mathrm{E}}$, the projection of the source star radius $R_{\mathrm{S}}$ onto the lens plane, measured in units of the Einstein radius $R_{\mathrm{E}}$. Taking a Sun-like source as reference, the parameter $z \equiv \beta / \rho$ is given by

$z=0.5\left(\frac{\beta}{0.1}\right)\left(\frac{t_{\mathrm{E}}}{0.1 \text { days }}\right)\left(\frac{R_{\odot}}{R_{\mathrm{S}}}\right)\left(\frac{1 / 2}{x}\right)\left(\frac{V}{200 \mathrm{~km} \mathrm{~s}^{-1}}\right)$.

Equation (14) shows that finite source effects are clearly measurable for $\beta=0.1$ and $t_{\mathrm{E}}=t_{\mathrm{E}}^{\star} \approx 0.1$ days. Moreover, they are always detectable for $\beta<0.1$ and $t_{\mathrm{E}}=t_{\mathrm{E}}^{\star}$ since Eq. (11) implies that $\beta t_{\mathrm{E}}^{\star}=$ const.

The Fisher analysis presented in Sect. 2 considers a point source. Can this assumption invalidate the error predictions of Fig. 3? The peak amplification influences $\sigma_{\pi_{\mathrm{E}}}$ via the relation $A_{\max } \simeq \beta^{-1}$. Therefore, finite source effects strongly modify the error predicted by a PSPL model if the maximum amplification of a finite source model, $A_{\max }^{\prime}=\sqrt{4+\rho^{2}} / \rho$ (Witt \& Mao 1994), is much smaller than $\beta^{-1}$, that is, $A_{\max }^{\prime} / A_{\max } \ll 1$. Because $A_{\max }^{\prime} / A_{\max } \simeq 2 z$, finite source effects strongly affect the error predictions only for $z \ll 1 / 2$ (Witt \& Mao 1994), which corresponds to $t_{\mathrm{E}} \ll 0.1$ days at $\beta=0.1$ for source stars not much bigger than the Sun. As Fig. 3 shows, these timescales are, however, of little importance since the error is already too big. For lower values of the impact parameter, $\beta<0.1$, and $t_{\mathrm{E}} \ll t_{\mathrm{E}}^{\star}$, the predictions are a priori strongly affected by finite source effects. However, since Fig. 3 shows that for $t_{\mathrm{E}} \ll t_{\mathrm{E}}^{\star}$, the sensitivity to $\pi_{\mathrm{E}}$ rapidly saturates when one reduces $\beta$, we do not expect important deviations even for these timescales.

In the case of giant-like source stars, $R_{\mathrm{S}} \gtrsim 10 R_{\odot}$, finite source effects are dominant since peak amplifications can be much lower than what is predicted by a PSPL model. Basically, one can think in terms of an effective impact parameter $\beta^{\prime}=$ $\beta(2 z)^{-1}$. For $R_{\mathrm{S}} \gtrsim 10 R_{\odot}$ and $t_{\mathrm{E}}=0.1$ days, one has $A_{\max }^{\prime} \simeq 1$ 
and $\beta^{\prime} \simeq 1$, and Fig. 3 suggests that $\sigma_{\pi_{\mathrm{E}}}$ would not guarantee a robust parallax detection. At $t_{\mathrm{E}}=1$ day, one gets $\beta^{\prime} \gtrsim 0.1$, and the parallax sensitivity is still marginal. Therefore, the finite source effects of giant-like stars seem to exclude robust measurements of $\pi_{\mathrm{E}}$ for Earth-mass lenses. However, larger sources generally yield smaller photometric errors $\sigma_{\mathrm{m}}$, and a parallax detection could still be feasible for closer lenses, i.e., $D_{\mathrm{L}} \lesssim 500 \mathrm{pc}$ or $x \lesssim 0.06$, and $R_{\mathrm{S}} \lesssim 10 R_{\odot}$ (see Eq. (14)).

\subsection{Earth umbra}

The Earth shadow can represent a significant limitation to the capabilities of a LEO satellite. It reduces the fraction $\eta$ of the orbital period available to observe the microlensed source star. We calculate the dependence of this fraction on the orbital radius $R$ and the event latitude $\lambda$ over the orbital plane,

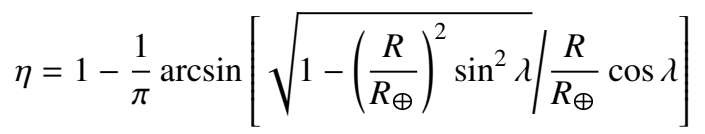

For $R=R_{\oplus}+550 \mathrm{~km}, \eta$ has a minimum value of $63 \%$ at $\lambda=0^{\circ}$, and it slowly increases to $79 \%$ for $\lambda=60^{\circ}$. For $\lambda \geq \lambda_{\star} \equiv \arcsin \left(R_{\oplus} / R\right) \approx 67^{\circ}$, the source star is always visible from the satellite. Consequently, even if the analysis of Sect. 4 is strictly valid for continuous observations $(\eta=1)$, Eq. (15) seems to indicate that Earth umbra does not invalidate its general conclusions when one tracks bulge stars. There is also some room to minimize the impact of the Earth shadow by adjusting the satellite orbital parameters. For an altitude of $1000 \mathrm{~km}$, for example, the angle $\lambda_{\star}$ decreases to $60^{\circ}$. Moreover, increasing satellite inclination above the equatorial plane can substantially reduce the shadow impact.

\section{Conclusions}

The present study employs a numerical Fisher matrix analysis to assess the feasibility of measuring microlens parallaxes by means of Earth orbit satellites. It extends the previous analytical analysis of Gould (2013) to shorter Einstein timescales. We predict that, at $A_{\max } \gtrsim 5-10$, a GSO satellite could detect microlens parallaxes for free-floating lenses with masses spanning from a fraction to a few dozen $M_{\text {Jup }}$ (providing their mass if $\theta_{\mathrm{E}}$ is also measured via finite source effects). It could also discover planets in tight orbits around very low-mass brown dwarfs. Moreover, at $\beta \lesssim 0.1-0.2$, a LEO satellite could discover free-floating objects ranging from terrestrial planets to super-Earths and ice giants. It could also detect, at $\beta \lesssim 0.05$, microlens parallaxes for Jupiter-mass free-floaters. Limitations to these results can be the strong requirements on the photometry $(0.01 \mathrm{mag}$ error with three-minute exposures), the effects of blending, and in case of a LEO satellite, the Earth umbra. It is useful to note that, even though this study adopts a single-lens model, we extrapolated its results to binary-lens events. Indeed, as far as a parallax effect is concerned, the fundamental quantity is the projected separation between the source star and the lens centre of mass. In planetary events this almost coincides with the host. What really differentiates the two kinds of events is that finite source effects are needed to measure $\theta_{\mathrm{E}}$ and estimate the lens mass: they are not routinely measured in single-lens events, while they are for binary ones.

Acknowledgements. F. M. is grateful to N. Cornuault, L. Pittau, and C. Ranc for the frequent and fruitful discussions. The authors acknowledge the support of PERSU Sorbonne Université and the Programme National de Planétologie.

\section{References}

Barry, R., Kruk, J., Anderson, J., et al. 2011, in SPIE Conf. Ser., 8151, 81510L Bennett, D. P., Batista, V., Bond, I. A., et al. 2014, ApJ, 785, 155 Gould, A. 2013, ApJ, 763, L35

Han, C., Jung, Y. K., Udalski, A., et al. 2013, ApJ, 778, 38

Hardy, S. J., \& Walker, M. A. 1995, MNRAS, 276, L79

Honma, M. 1999, ApJ, 517, L35

Joergens, V., Bonnefoy, M., Liu, Y., et al. 2013, A\&A, 558, L7

Luhman, K. L., Adame, L., D’Alessio, P., et al. 2005a, ApJ, 635, L93

Luhman, K. L., D’Alessio, P., Calvet, N., et al. 2005b, ApJ, 620, L51

Sellentin, E., Quartin, M., \& Amendola, L. 2014, MNRAS, 441, 1831

Spergel, D., Gehrels, N., Baltay, C., et al. 2015, ArXiv e-prints [arXiv: 1503.03757]

Vallisneri, M. 2008, Phys. Rev. D, 77, 042001

Witt, H. J., \& Mao, S. 1994, ApJ, 430, 505 